aspects includes a lengthy account of the arguments on whether Black and White in the U.S. differ genetically in mean IQ. The authors conclude there is no evidence for this. It also includes a discussion of any possible dysgenic trends of differential fertility by social class and intelligence. The authors conclude, probably correctly, that there is no reason to assume any dysgenic trends at present, and might have added that differences in family size by social class in the most recent United States and European censuses have largely disappeared, though there are still urban-rural differences.

The section on positive eugenics is perhaps rather old fashioned and tends to stress the difficulties of aim and method. Most of these difficulties disappear if the approach is at the level of the individual parents. These have the moral duty to consider the probable genetic potential of their children for healthy development of mind and body in deciding the size of their families. The task of the human geneticist is to spread knowledge of human genetics so that parents will themselves make informed decisions, rather than to direct the parents along certain lines of action. This book will make a valuable contribution to such instruction.

Cedric Carter

\section{Early Diagnosis and Prevention of Genetic Diseases.}

Boerhaave Series for Postgraduate Medical Education, No. 11. Edited by L. N. Went, Chr. Vermeij-Keers, and A. G. J. M. Van Der Linden. (Pp. 160; Figures + Tables.) Leiden, The Netherlands: Leiden University Press. 1975.

This volume includes the content of a postgraduate course in the Boerhaave Series organised by The Faculty of Medicine, University of Leiden. There are 15 contributors: the majority, a proportion of whom are theologians, are from the Netherlands, but others are from the United States and England. Fourteen formal papers are included with a very short discussion at the end of each. The final chapter, on the ethical aspects of prevention, is entirely in the form of a discussion and includes some speakers, presumably from the audience, who are not listed as contributors.

The formal presentations, some more detailed than others, cover Huntington's chorea, dystrophia myotonica, retinoblastoma, phenylketonuria, cystic fibrosis, and the sphingolipidoses. These early chapters, which also include neural tube defects and the detection of carriers of haemophilia, lead into a more broadly based section on prenatal diagnosis, possible methods of detection of genetically determined disease, both in the family and in the community, and genetic counselling.

Apart from the problems associated with publication of symposia of this type, this volume seems a usefu I contribution to the literature at present because the various presentations do highlight some of the dilemmas in human genetics. These are not always comparable with those encountered in clinical of? community medicine as currently practised and noveg solutions are, therefore, required. The implications are substantial and it will be wise to seek advice fromf sections of society other than the scientific. It is $\underset{\Omega}{\mathbb{D}}$ therefore, encouraging to find theologians included here though it is clear from the final discussion that there was also a great deal of ethical variation of opinion!

Gerald Corneycu

Methodology in Medical Genetics. An Introduction to Statistical Methods.

By Alan E. H. Emery. (Pp. 145, illustrated. £6.50.) Edinburgh: Churchill Livingstone. 1976.

Reading this book proved to be an enjoyable and rewarding experience for the reviewer, who learnt $a^{?}$ considerable amount in the process. Its value lies not only in its compact size and practical approach but from the fact that it brings together the majority of methods of analysis that workers in medical genetics require in handling their data, methods thate are often hard to find in textbooks of either statistics $V$ or genetics. Throughout the book worked exampleso are given, based on practical problems and data, which add greatly to its value. No extensive knowledge of mathematics is assumed, which is a distinct advantage to those workers in the field (one suspects stillo the majority!) who do not have a natural inclination in this direction.

The book opens appropriately with an explanation and derivation of the Hardy-Weinberg Equilibrium and then illustrates the estimation of gene frequencies for the different types of inheritance and for multiplez alleles. The fully worked examples are particularlyo clear and helpful. The chapter on the genetic structure of populations is of necessity limited, but the esti-i mation of consanguinity by different approaches is well discussed and illustrated, as is the analysis of $\mathrm{O}$ fitness and its relation to the frequency of mutation. $A^{-}$ table gives useful data on this in various X-linkedo disorders.

Segregation analysis receives a separate chapter,, with particular emphasis on the problems of auto- $N$ somal recessive inheritance. Full tables are given for the use of Li and Mantel's 'singles' method in addition ${ }^{\omega}$ to the $a$ priori and maximum likelihood methods. Multifactorial inheritance is discussed in relation to heritability and twin studies, and the limitations of heritability are perhaps not sufficiently emphasised; this is apparent from the table of heritability estimates which shows early onset of diabetes to be much greater than that of late onset, contrasting with recent $\frac{\stackrel{\oplus}{\Phi}}{\sigma}$ 